\title{
Sociomaterial analysis of Music Notation Lessons: Virtual work and digital materialities
}

\author{
Demosthenes Akoumianakis \\ Department of Informatics Engineering, Technological Education Institution of Crete (TEI Crete) \\ Heraklion, Crete, Greece
}

\begin{abstract}
The present research rests and elaborates on sociomaterial aspects of virtual practices, as manifested through distributed and collaborative work. This is approached through an interpretive case study of music notation lessons (MNLs) using the DIAMOUSES system. Our empirical data suggest that sociomateriality shifts the focus of designing interactive technologies from mere considerations of digital manifestation (i.e., forms of representation) towards explicit accounts of the representational practices (i.e., the particular material properties of these forms) and the quality attributes to be embedded in technology.
\end{abstract}

Keywords-Virtual work, affordances, design qualities, case study research

\section{INTRODUCTION}

Sociomateriality is a recent construct aiming to explain the relationship between the social and the material across work settings and organizational contexts. Such an intriguing challenge turns out to be difficult due to the lack of sufficient ground to anchor the varieties of 'agencies' implicated in the social and material realities of organizational life. Recent management and organization science scholarship seeks for theoretical insights by recapitulating constructs such as social practice [1] [3], affordances [2] and virtual team work [4] [26]. Information Systems (IS) researchers [5] [6] [9] explore and classify facets of sociomateriality. The Computer-Supported Cooperative Work (CSCW) community is also engaged in debates about the configurations of the social and material [7], the way in which new digital technologies establish new materialities [8] and the techniques which may offer useful insights [10]. In most of these studies, sociomateriality rests on the recognition that technologies, people and organizations are constitutively entangled, rather than a priori self-contained entities, distinctively and separately studied [3]. Nevertheless, this notion of 'entanglement' may not offer an appropriate lens for design. Instead, as Leonardi [4] [22] convincingly argues 'imbrications' may serve better design-oriented thinking, especially in fields such Human-Computer Interaction ( $\mathrm{HCI}$ ) and $\mathrm{CSCW}$, which constitute the venues motivating the present research.

The present work aims to contribute to the on-going debate about digital materiality [8] and the way in which it is crafted and implicated into social / organizational practices. The normative perspective adopted is that digital materiality anchors new practices which do not follow inexorably from the material features of established technologies; instead, they are improvised on the basis of old practices that work differently in new technological circumstances and frequently lead to changes in social configurations [11], [12]. In this vein, our empirical ground stems from on-going work in collaborative music practicing. Such a focus should complement other research works making claims about how social media, networking platforms, blogging and microblogging services drive cultural shifts in the way people socialize online [13], use language [14] and organize offline arrangements, such as vacations and Network Music Performance (NMP) [15], [16]. The present work is seen as complementary to these efforts in so far as it offers insights into intrinsic properties of technology that bring about, enable or constrain these changes to the volume and extent that justifies cultural shifts or changes in social practice. Recent scholarly works acknowledge the need for better understanding the IT artefact and call for challenging the prolonged concerns with immaterial properties of information systems [17], [8]. They also point to a direction for research to advance a theory of digital materiality, thereby revisiting our understanding of computer-mediated artefacts and the ways in which they invoke material concerns of whatever practice is engaged.

In light of the above the specific aim of this paper is twofold. Firstly, it sets out to explore and untangle features embedded in digital technologies such as abstraction, modularity, persistence, sharing, connectivity, etc., whose presence or absence may determine what people do (individually or collectively) and the respective enacted phenomena. Secondly, it seeks to establish a preliminary empirical ground by examining these features in a particular virtual work setting - that of online music notation lessons (MNLs) - and the digital artefacts involved. Phrased differently, our goal is to investigate sociomaterial aspects of computer-mediated music practicing and the intrinsic properties, such as representations, tools and software quality attributes, that shape and augment the practice in virtual settings. The approach builds on two threads of research; on the one hand, it reflects upon recent empirical findings about online music ensembles [10] and on the other hand, it transforms these findings into design proposals anchoring distributed music making as virtual work [18]. The main contributions are to be found not so much in the theoretical treatment of the concepts involved, as on the (designoriented) mechanics that may determine what is possible under certain circumstances and the methodological challenges confronting HCI researchers interested in understanding how new technologies enable or constrain what people do online. 
The rest of the paper is structured as follows. The next section motivates the present work by reviewing relevant scholarships and establishing the research focus. Then, we present the methodology for studying a specific NMP scenario, namely music notation lessons. The approach entails revisiting an existing research setting through an interpretive case study and instruments that bring to the surface sociomaterial considerations. The paper is concluded with an outline of implications and ongoing and future research.

\section{THEORETICAL MOTIVATION AND RELATED WORK}

Despite wide acknowledgements of the new primary beneficiaries established by new technologies [19] [20], it is not yet clear exactly what may be the features embodied in these technologies (material) and how these implicate novel (social) practices. This is the rationale for bringing sociomateriality at the forefront to assess not only the concept's theoretical underpinnings but also some of its implications for designers. To this effect and by elaborating on different theoretical literatures, such as digital materiality [17] [8], imbrications [22] [23], practice theory [21], remediation [24] and digital assemblages [25], the present research seeks to establish its theoretical footing.

Attempting a broad classification, it may be claimed that current thinking on sociomateriality emphasizes two metaphors coined by the 'entanglement' of agencies and the 'imbrication' of structures. Both perspectives share common ground, but they lead to slightly different insights. For instance, they both advocate the notion of 'affordances', initially introduced by ecological physiologist James Gibson [31], to anchor the material grounds of either computermediated work or daily activities [2], [22]. However, entanglement favours certain (perceivable) product-oriented affordances, while the imbrications perspective emphasizes design-embedded affordances (which may not be easily perceived at first sight). It is also common to relate sociomateriality with remediation [24] and the notion of digital assemblages [25], but there are various forms of remediation that may not implicate new digital assemblages.

In such a broad setting, our current effort adopts a design -oriented perspective focusing on digital artefacts, their transformative capacity as well as the way in which they are inscribed into different technological configurations and settings. As artefacts we consider not only the digital manifestation of objects but also the material qualities through which these objects become embedded into organizational life. This perspective allows us to theorize about the 'cultural' history of artefacts in terms of mediaspecific representations and affordances as well as to foresee the tactics through which they are remediated to ascribe (new) material agency to certain technologies.

\section{A. Affordances and virtual work}

The concept of affordances was introduced by Gibson to coin opportunities for perception and action offered by the environment to an organism, whether human or not [31]. Norman [32] suggested a slightly different interpretation focusing on '...perceived and actual properties of the thing, primarily those fundamental properties that determine just how the thing could possibly be used (p.9)'. In subsequent works, Norman [33] acknowledged misuse of the term and made a further clarification, stating that '.. it is very important to distinguish real from perceived affordances' and that '... design is about both, but the perceived affordances are what determine usability...' (p. 123). Despite differences in orientation, Gibson and Norman recognize that material properties of physical objects determine the actions each object invites for or constrains. Accordingly, agency is critical since possibilities of action are not given. In a slightly different vein, Gaver [34] by-passes the issue of agency claiming that it is the design that suggests an affordance and therefore, affordances 'can be designed and analyzed in their own terms.' (p. 81). According to this view, affordances of computer-mediated environments can be related to design qualities such as abstraction, translucence, interoperability, connectivity and plasticity. Then, it may be argued that it is the presence or absence but also the intertwining of these design qualities that enables or constrains use of computermediated artifacts as well as the part of such use that is retained and made available for exploration. Arguably, this is useful but in need of further refinement if it is to provide a basis for designing systems.

Following a different strand and building on semiotics, Bailey, Leonardi \& Barley [26] have advanced a conceptual lens that is useful for understanding virtual work in terms of the intrinsic properties of computer-mediated practices and affordances. The authors distinguish between digitization and virtuality by anchoring their implications. Thus, they claim that digitization implicates computer-based representations of physical phenomena, while virtuality occurs when digital representations stand for, and in some cases completely substitute for, the physical objects, processes, or people they represent. Building on this distinction, the authors qualify computer-mediated work as virtual teams, remote control or simulations, while in each case they classify the human activities involved as operations with or on, and within or through representations. By this account Bailey, Leonardi \& Barley resurface the notion of affordances to assess how certain representations (and the practices they implicate) obtain material features through the way in which they become embedded into whatever technology is at hand.

\section{B. Remediation}

The concept of remediation was only recently introduced in the information systems literature in an effort to assess how certain practices are aligned and re-aligned to certain media. Lanzara [24] defines remediation as a migration of an assemblage of embedded agencies established in a certain medium to a new assemblage in a new or multiple media. Lanzara's [24] also acknowledges that 'the more deeply embedded is the practice in a specific medium, the greater the amount of restructuring involved in the migration to a different medium'. At core what is called for is an assessment of the degree of embeddedness of practices into 
certain media through accounts of intrinsic constructs such as representation of meaning, means of transmission and synergistic use of media. There are practices, such as painting and music composition that tend to avoid detail and value abstraction. They also tend to embrace ambiguity as enabling richness of meaning and bring into play our intuition and imagination that determine how the practice is enacted. These practices - frequently referred to as creative practices - explore visual, spatial, textural and audio representations that afford abstraction, rich meanings and interpretation. In contrast, there are technical practices that aim to eliminate ambiguity, seek for certainty and pursue correctness, completeness and detail. They rely on formalism, symbolic representations and logical reasoning.

It then stands to ask 'How are practices remediated (using technology) and what effects such remediation may bring about?' Clearly, new technologies and media can have variable effects on practices. For instance, [14] examines how a typographic convention, the hashtag, operates as a linguistic marker, thus rendering the language searchable and driving a cultural shift for electronic discourse from online conversation to such 'searchable talk'. Similarly, Dourish and Mazmanian [8] describe how digital photography and the material constitution of digital images allow for forms of manipulation quite different from those that film photography affords. These examples are indicative not only of the scope of remediation but also of its implications that may vary from mere improvements in the conduct of practices to establishing totally new practices whose domain of discourse is anchored on new grounds [16].

\section{Practice theories}

Attempting to establish some sort of benchmarks or thresholds to understand intended and unintended effects of remediation, practice-oriented thinking and theories [21], [27] - despite their different orientations - offer a potentially useful roadmap. Of particular relevance to the present work is the notion of the practice lens [27], [28] as a metaphor for analytical inquiries. According to the practice lens, technologies can be seen as prerequisites for particular outcomes but the existence of prerequisites does not determine the outcome. Thus, by appropriating features inscribed in technology it is possible to enact new structures which were not initially foreseen during the development of the technology. Such a line of thinking is consistent with Pickering's concept of 'temporarily emergent' activities [30] and Suchman's notion of 'socio-materiality' of practice [7].

For our purposes, practice-based theorizing translates to a rather distinct line of argumentation. Specifically, it is argued that although functionality embodied in technological artefacts is clearly important, it is not likely to be, by itself, the sole determinant of how the artefact is used in practice. Instead, the presence or absence of non-functional design qualities embedded in technology can catalyze and determine technology use [29]. This is evidenced when examining what constitute embedded qualities in established and emerging technological paradigms. Specifically, technological transitions such as the evolution from the WWW, to Web 2.0 and Social Semantic Web as well as the associated practices (i.e., from device-dependent to device independent mark-ups, from authoring to collaborative editing and from consuming information to social networking and end-user content creation) can be explained by the increasing embeddedness of qualities, such as abstraction, portability and interoperability, in technological artefacts and tools [10].

\section{Consolidation and reasearch questions}

Attempting to synthesize the discussion thus far, it is argued that three issues stand out very prominently. Firstly, practices comprise activities on digital artifacts, which in turn, are representations of objects (human and non-human). Secondly, the affordances of these representations determine by and large the possibilities and effects of what is possible in a certain medium as well as the viability of remediation (i.e., shifting the practice form a certain medium to another). Thirdly, the material concerns invoked by digital technologies stem (at least in part) from the presence or absence of quality attributes that ascribe certain affordances to artifacts embedded in technology. Based on the above, several interesting questions stand out very promptly with regards to: (a) How are social and material agencies configured and enacted in a virtual setting? (b) What is the unit of analysis through which designers can gain insight to material agency, and (c) Does this focus on sociomateriality improve upon current design-oriented thinking and activities in the fields of HCI and CSCW?

\section{METHODOLOGY}

To shed light to these concerns, this section elaborates on an interpretive case study of collaborative MNLs. MNLs in the broader context of NMP present an interesting and challenging case that fosters the migration of an assemblage of embedded agencies established through sensory-based media to a new digital assemblage amenable to virtual work. Arguably, sociomaterial concerns constitute a core theme in pursuing pathways for such migration. Our case rests on an existing system, called DIAMOUSES [35]. DIAMOUSES offers an appropriate research setting to explore sociomateriality for several reasons. Firstly, it was designed to support MNLs as well as other NMP scenarios. Secondly, it was the first system to promote a separation of concerns; one component undertakes community management functions while another one implements music co-practicing [16]. This suits our purposes as it makes it easier to locate and 'trace' community- and practice-oriented features. Finally, DIAMOUSES could be easily modified to facilitate virtual ethnographic analysis of online music ensembles, which is one of the methods used to solicit data.

\section{A. The research setting: DIAMOUSES notation lessons}

DIAMOUSES is a system for NMP which demands a specialized set up in terms of equipment and software. Figure 1 depicts a typical DIAMOUSES configuration which can serve a variety of NMP scenarios ranging from rehearsals, improvising and learning. A MNL with DIAMOUSES represents a case of reconstructing online widely accepted practices based on established music constructs. 


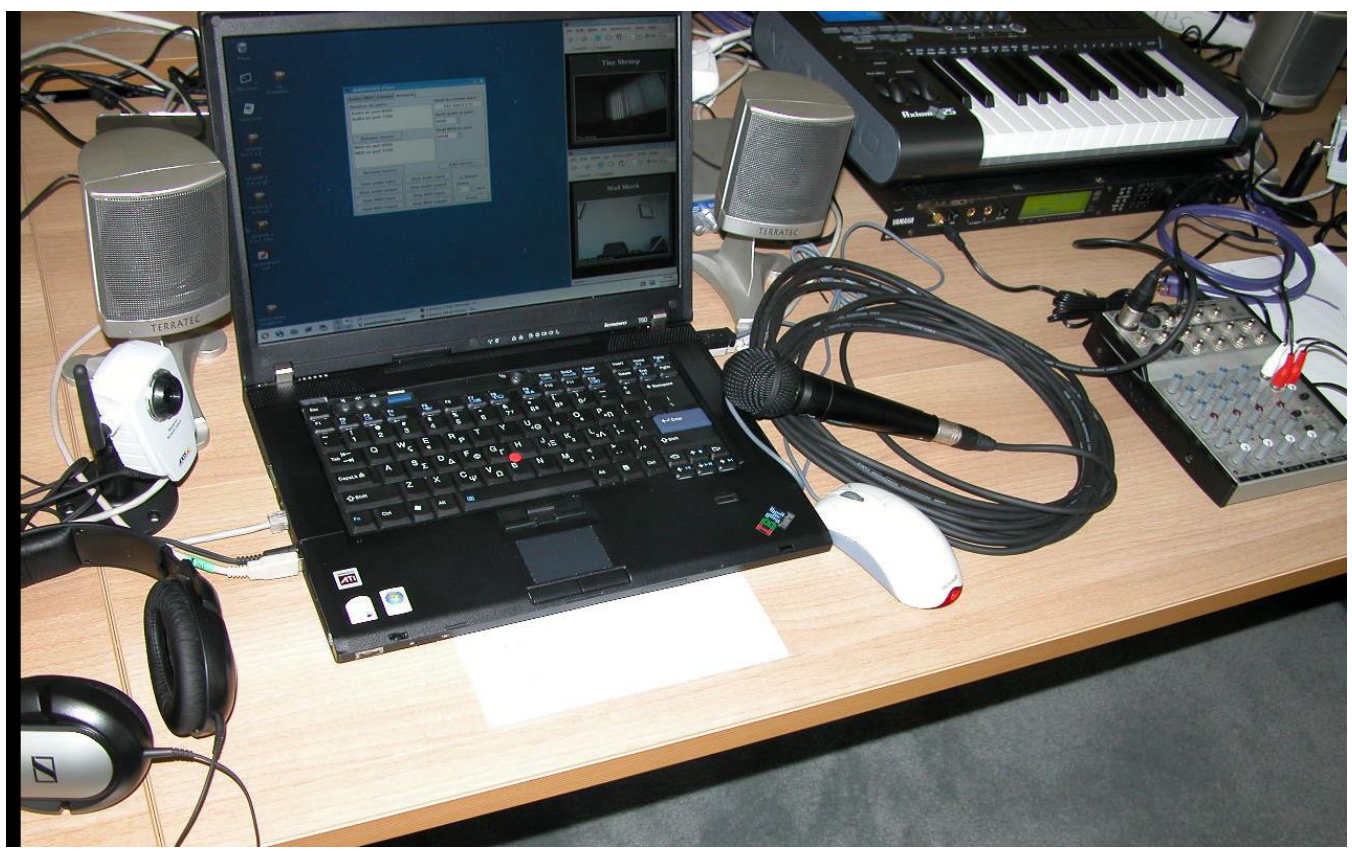

Figure 1: A typical DIAMOUSES configuration
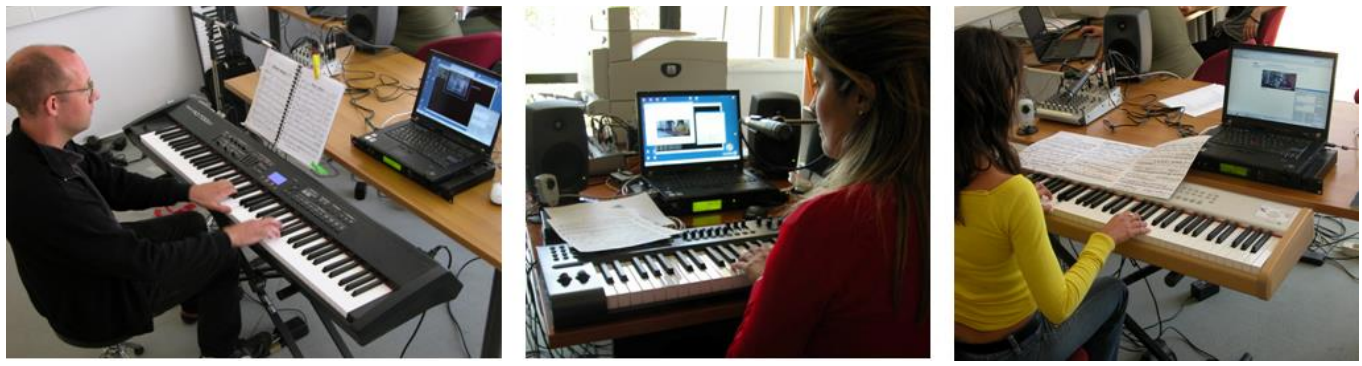

Figure 2: DIAMOUSES piano lesson

To conduct MNLs with DIAMOUSES, moderators (or music theory tutors) prepare shared music materials (i.e., score, recordings, videos), schedule and organize the music lesson and invite participants. There are two prerequisite for taking part in MNLs. The first is the users' acceptance of the moderator's invitation, which is followed up by registration to a virtual 'room' containing the shared material of the lesson. Registration is a two-stage process where participants first become members of the community (by building their music profile) and then register to 'rooms'. The second prerequisite entails downloading the dedicated practicespecific software suite, which allows members to engage synchronously in the micro-negotiations of a specific music lesson. This toolkit provides a virtual space for participants to access shared music materials and negotiate them against their personal technical virtuosity. The scenario addressed in our experiment represents a multi-site engagement in a piano lesson with one moderator and several participants (see Figure 2).

\section{B. Instruments and data collection}

In order to set the focus on social and material aspects of MNL, it is compelling to define each constituent and identify possible measurable features of each. In our current analysis, the 'social' constituent is conceived as the online ensemble that is brought together to take part in a MNL. The agency of online ensembles is traceable by account of 'cultural' artifacts of practice. These are broadly defined as the digital remains that reveal individual or collective activity taking place either prior, during or following a MNL.

The 'material' constituent coins the digital representations embedded in technology which drive the technology's performative capacity. These can be assessed by examining designated quality attributes that enable or constrain use in any particular setting. This gives rise to the theoretical scaffold summarized in Figure 3. Thus, our normative perspective is that social structures such as online ensembles operate with, on or within and through representations embedded in technology by ways which determine (i.e., enable or constrain) certain uses. Then, such line of thinking can inform the design of an interviewing instrument which will comprise questions on cultural artefacts and design qualities.

To solicit information on what may be the range of plausible cultural artefacts, available scholarship was 
consulted pointing out generic artefacts [20] (i.e., user profiles, expressing opinion \& communication, finding \& sharing information, establishing connections) as well as practice-specific [16] (i.e., organizing and performing own work, contributing to the shared practice agenda, coordinating / aligning with others and making sense of what is expected, aligning online and offline activities). In terms of quality attributes, our analysis relies on earlier works [29] indicating the role of certain attributes such as abstraction, portability, translucence, information and social connectivity. For each quality attribute a number of screening criteria were established to facilitate an interpretation of the quality attribute in the users' language.

Thus, a data collection strategy was devised comprising qualitative interview data and digital traces highlighting online activities in the course of virtual ethnographic studies. Interviews were scheduled and carried out after the virtual ethnography of an online music ensemble with the researcher becoming actively involved in the practice of an online MNL. Further details on the instruments devised, the screening criteria, the specific questions, the data compiled and the data analysis methods have been elaborated elsewhere [10].

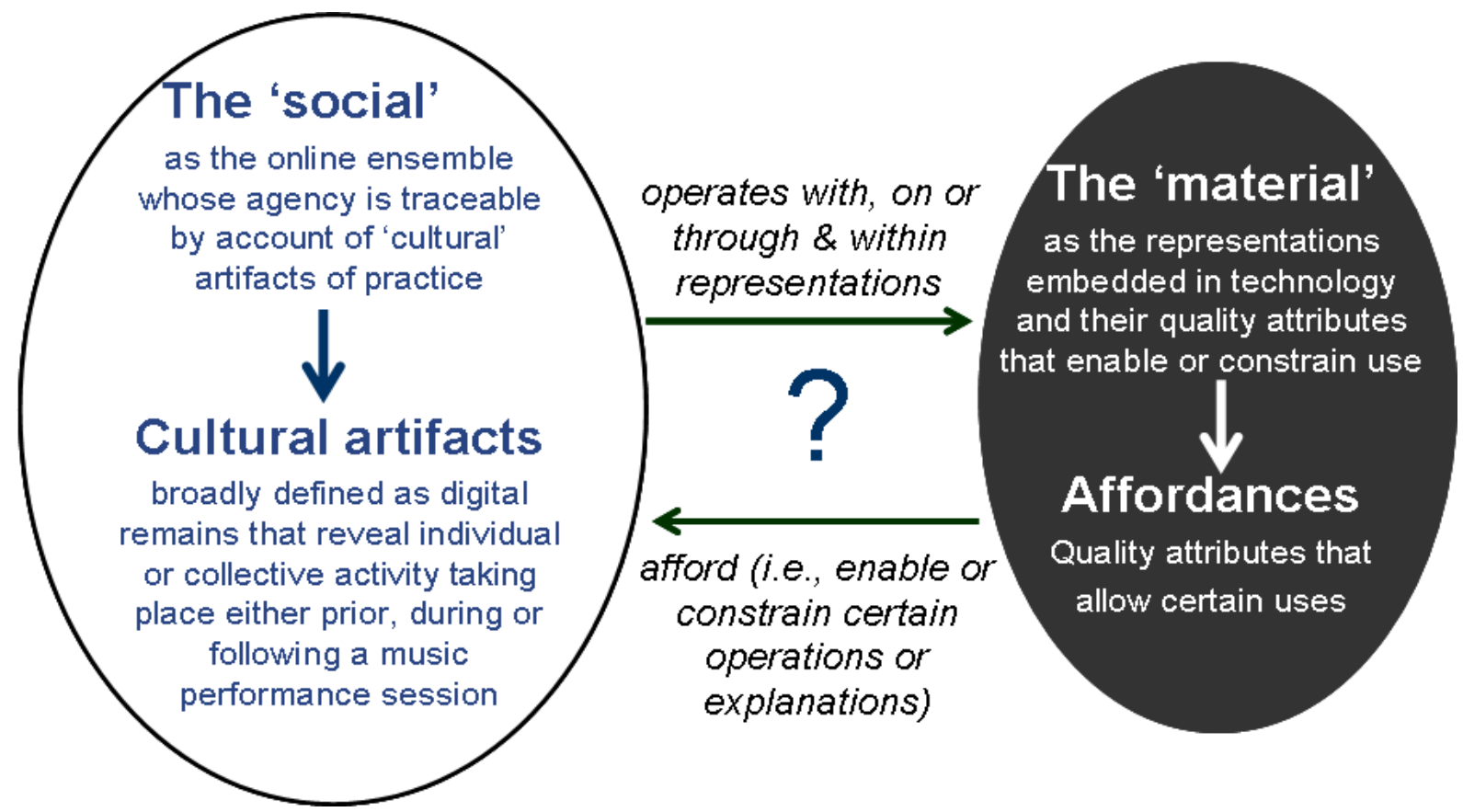

Figure 3: Theoretical scaffold

\section{Summary of findings}

Table I consolidates participants' responses to questions intended to unfold generic and practice-specific cultural artefacts and the virtual settlements in which their traces are retained. All participants confirmed that online ensembles emerge and sustain their function through the members' recurrent interactions in two separate but interrelated virtual spaces, namely the community management system (coined by the 'LR' code in Table I) and the music toolkit for coengagement in synchronous MNLs (coded as 'MT' in Table I).

With respect to generic cultural artefacts participants acknowledged all those revealed by earlier studies [20], namely user profiling, tools for expressing opinion and communicating, finding and searching for information and establishing connections. As for practice-specific cultural artefacts (shaded rows), participants identified a wide variety, broadly classified in three sub-categories/sub-codes: (a) performative artefacts used to execute a shared agenda (b) coordinative artefacts that facilitate coordination and distributed organizing in the course of executing the agenda and (c) collective artefacts of historical value that reveal the outcome of the online music ensemble. As shown in Table I, most of these artefacts are traceable in a single settlement - in most cases the music toolkit - with the exception of the music score and the supporting documentation that were deemed as having cross-settlement traces.

In the category of practice-oriented performative artefacts the score, as means for representing music (through XML scripts in the LR community management system or visual representations in the Music Toolkit), was the most frequently cited cultural artefact. From the participants' responses, it turns out that the score, in addition to being a learning material, it also serves as a boundary object intertwining between the online and offline setting, thus serving the purpose of structuring (and re-structuring) unknown contexts and/or actions and assigning them with meaning. In contrast to performative artefacts, practice-oriented coordinative artefacts were considered to be those that facilitate micro-negotiations during a MNL. In this category participants identified the metronome, the floor manager and objects for controlling remote user's performance. The final category of practiceoriented cultural artefacts includes those of historical value that 
consolidate a collaborative engagement. All respondents identified the recorded performance (or recorded audio signal) as a socially constructed cultural artefact offering longstanding and traceable evidence of the ensemble's existence. It can therefore be concluded that the remains of a virtual ensemble engaged in MNLs comprise on the one hand the dynamics of collaboration during the lesson and on the other hand, the 'packaged' outcome codified in an audio format.

Assessment of the design qualities inscribed in technology and the extent to which they enable or constrain use turned out to be more challenging, as specific methods to anchor such features are lacking. As a result, heuristic assessment was recruited to provide the required evidence. Table II summarizes the results and confirms that DIAMOUSES exhibits several limitations and shortcomings. The first observation is the lack of any evidence for portability, abstraction, translucence and plasticity of the cultural artefacts coined as generic. This is not surprising as DIAMOUSES was not conceived or designed as social web site (in the sense suggested in [20]). In terms of digital manifestation (i.e., form), the system's focus was on GUI toolkit enhancements and CSCW oriented inscriptions for replicating domain-specific artefacts such as the score and synchronizing client applications. This constrained the range of digital representations implicated in practice and imposed strict schemes for binding across representations. As for the material features of these representations, the design of DIAMOUSES failed to acknowledge the properties that condition the forms of social action invoked by the embedded digital representations. This is derived from the weak support (i.e., Xw in Table II) evidenced by our users. More detailed analysis of participants' responses (see [10]) reveals loose accountability for explicit and implicit boundaries and lack of boundary spanning mechanisms. Similarly, the social protocols for co-engaging in practice are undermined and underserved.

Having outlined the limitations, it is important to notice that at the time of designing DIAMOUSES, such concerns were not widely shared or easily accommodated in technical specifications. Subsequent extensions in web services and standards, the increasing availability of public APIs for interoperability and the trend towards virtualization and cloud services, have created new opportunities and reset the research agenda.

TABLE I. SUMMARY OF FINDINGS ON CULTURAL ARTIFACTS

\begin{tabular}{|c|c|c|c|}
\hline \multirow[b]{2}{*}{ Question } & \multirow[b]{2}{*}{ Artifact } & \multicolumn{2}{|c|}{ Virtual settlement } \\
\hline & & LR & MT \\
\hline Objects/artifacts intended to register and obtain roles & Custom registration system & $\mathrm{x}$ & \\
\hline \multirow{3}{*}{ Tools for commenting \& communicating } & Forum & $\mathrm{x}$ & \\
\hline & Chat & & $\mathrm{x}$ \\
\hline & Camera-based surveillance & & $\mathrm{X}$ \\
\hline $\begin{array}{l}\text { Objects / artifacts for locating codified information or uploading user-created } \\
\text { content }\end{array}$ & $\begin{array}{l}\text { Liferay room, dedicated information templates } \\
\text { and custom portlets }\end{array}$ & $\mathrm{x}$ & \\
\hline \multirow{2}{*}{ Tools for building and maintaining social ties } & Extend invitation (tutor) & $\mathrm{X}$ & \\
\hline & Accept invitation ( participants) & $\mathrm{X}$ & \\
\hline \multirow{2}{*}{$\begin{array}{l}\text { Objects/artifacts whose manipulation results in traceable digital remains of } \\
\text { my own performance }\end{array}$} & Music score as XML script & $\mathrm{x}$ & \\
\hline & Music score as Interactive graphical object & & $\mathrm{x}$ \\
\hline \multirow{2}{*}{ Objects/artifacts that tell me what to do in relation to others } & Synchronous chat & & $\mathrm{x}$ \\
\hline & Social proxies on music score & & $\mathrm{x}$ \\
\hline \multirow[b]{2}{*}{ Objects/artifacts for making sense of the joint agenda and what is expected } & Metronome & & $\mathrm{x}$ \\
\hline & $\begin{array}{l}\text { Floor manager and states i.e., occupied vs. } \\
\text { released }\end{array}$ & & $\mathrm{x}$ \\
\hline \multirow{2}{*}{ Objects/artifacts for understanding what others are doing } & Remote audio signals (output) & & $\mathrm{x}$ \\
\hline & Mute (on/off) & & $\mathrm{X}$ \\
\hline \multirow{2}{*}{ Objects/artifacts for online \& offline alignment } & Local audio stream (input) & & $\bar{x}$ \\
\hline & Mute (on/off) & & $\mathrm{x}$ \\
\hline \multirow{2}{*}{ Objects/artifacts for collective performance } & Mixed recorded performance & $\mathrm{x}$ & \\
\hline & Supporting documentation & $\mathrm{x}$ & $\mathrm{x}$ \\
\hline
\end{tabular}

TABLE II. DESIGN QUALITIES INSCRIBED IN TECHNOLOGY

\begin{tabular}{|c|c|c|c|c|c|c|c|c|}
\hline & \multirow[b]{2}{*}{ PR } & \multirow[b]{2}{*}{$\mathbf{A B}$} & \multirow[b]{2}{*}{ TR } & \multirow[b]{2}{*}{ Pl } & \multirow[b]{2}{*}{ IC } & \multirow[b]{2}{*}{ SC } & \multicolumn{2}{|c|}{ Virtual settlement } \\
\hline & & & & & & & LR & MT \\
\hline User profiles & & & & & & $\mathrm{X}$ & $\mathrm{X}$ & \\
\hline Expressing opinion \& com/tion & & & & & $X$ & & $\mathrm{X}$ & $\mathrm{X}$ \\
\hline Finding and searching information & & & & & $\mathrm{x}$ & & $\mathrm{x}$ & $\mathrm{X}$ \\
\hline Establishing connections & & & & & $\mathrm{X}$ & $\mathrm{X}$ & $\mathrm{X}$ & \\
\hline Music score & $x$ & $x$ & Xw & Xw & $\mathrm{X}$ & $x$ & $x$ & $\mathrm{X}$ \\
\hline Metronome & & & $X_{w}$ & & Xw & & & $\mathrm{x}$ \\
\hline Floor manager & & & $\mathrm{X}$ & & Xw & & & $X$ \\
\hline Remote performance controls & & & & & Xw & & & $\mathrm{x}$ \\
\hline Recorded performance & & & & & & $X$ & $x$ & \\
\hline
\end{tabular}




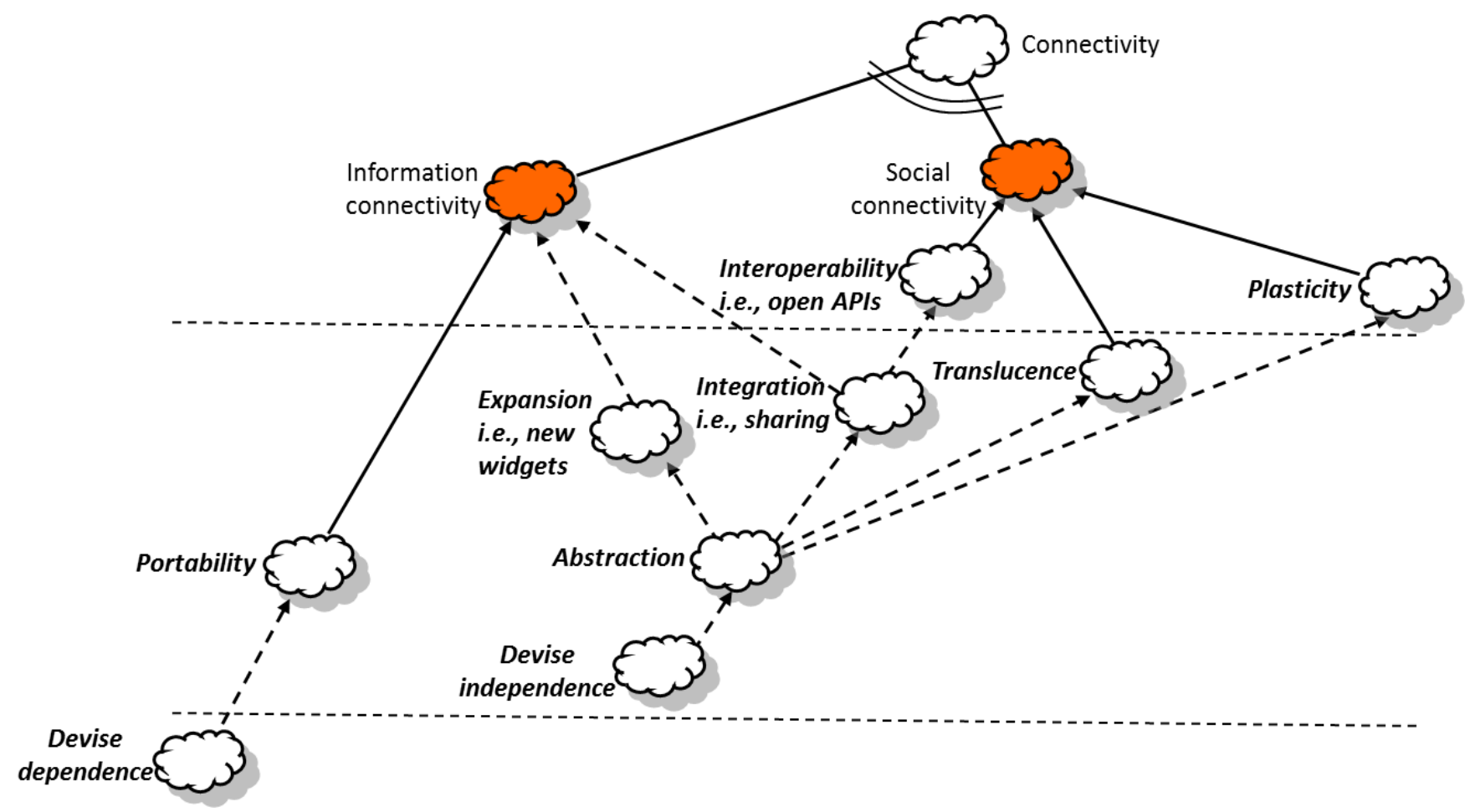

Figure 4: Inscribing design qualities into code

\section{DESIGN IMPLICATIONS \& DISCUSSION}

\section{A. Revisiting the research questions}

The analysis presented thus far, allows us to reformulate our understanding of sociomateriality in the context of online MNLs. Thus, it is claimed that online music ensembles can be conceived as enacted cyber-structures whose agency in virtual space stems from the members' capacity to operate either with, on or through and within digital representations embedded in technologies. At the material level, such capacity can be further qualified not so much by the medium-specific affordances after all, in the virtual setting everything is digital - as by the design affordances of the representations. It is these affordances and the presence or absence of quality attributes that enable or constrain not only what users can do, but also what is retained of their activities in the form of digital traces. In light of the above, it is worth revisiting the three questions relevant to present work (see section II.D).

With regards to the enactment of agencies, it is argued that social structures such as online music ensembles exercise control of representations embedded in technology to facilitate designated representational practices, such as MNLs. Material agency is enacted to anchor the technology's performative capacity which is determined by embedded design affordances and the inscriptions for portability, abstraction, translucence, portability, connectivity, etc.

As for the unit for analyzing material agency, our case study points to (a) digital traces and remains as the locus of cultural artifacts (see theoretical scaffold introduced in Figure 3) and (b) the transformative capacity stemming from the presence or absence of designated quality attributes. Through this lens, it is possible to dis-entangle the 'social' and the 'material' constituents of a digital assemblage, so as to reconfigure material capacities thereby creating new capabilities for action. According to this view technologies can be taken apart and reassembled so as to facilitate new (and improved) social agency. Similarly, social agency is enacted in response to whatever material concerns are invoked by technical configurations.

Finally, in terms of sociomateriality's contribution to design-oriented thinking in the fields of HCI and CSCW (third research question), it is fair to conclude that our analysis favors the notion of 'imbrication' as a guide to designing digital artifacts and novel virtualities. Nevertheless, it should be acknowledged that using 'imbrication' as a lens for design requires further attention and detailed treatment which forms a part of our ongoing work [18].

\section{B. Implications for design}

Without underestimating the variety of issues pending attention, it is claimed that the material described in this paper raises implications for designers of computer-mediated representational practices. The most prominent is that it brings to the forefront the need to address (non-functional) quality attributes as first class design properties. To grasp the point one may recall briefly the pathway driving the evolution towards Web 2.0 and the Semantic Web. Specifically, the compelling need for portability in the initial WWW era was accommodated by virtual machine environments, Graphical User Interface toolkits and device-dependent mark-up. The next wave targeted abstraction and interoperability and was enabled by device independence and public Application Programming Interfaces. As a result web site authoring practices made way for blogging and collaborative editing practices, while end users became content generators (across different micro- 
context such as blogging platforms, social networking services and other virtual settlements) from mere information consumers. In the current computing era, it stands to argue that technologies broadly classified under the Web 2.0 paradigm can be assessed in relation to a set of common provisions which inscribe in code certain degrees of connectivity. Schematically this is depicted in Figure 4 where design qualities (presented as clouds) intertwine to facilitate information and social connectivity. The point to be underlined is the variety of digital materialities that emerge as a result of different configurations of the designated qualities and the specific ways in which they become intertwined in code. Phrased differently, it may be argued that at any point in time there is a variety of digital materialities emerging from design commitments to different strategies that prevail within a certain computing paradigm. Thus, what is of interest is not so much the particular mix of quality attributes, but the extent to which such a mix and the ways in which it may be supported (i.e., open APIs, social aggregation services or any other kind or form of interoperability) leads to new capabilities and social practices.

\section{SUMMARY \& CONCLUSION}

The present research is a step towards understanding how technologies are enacted in practice and the material concerns invoked by the presence or absence of designated quality attributes. It turns out that such qualities define a space of opportunities that anchor what can be done with the technology at hand. It is also evident that there is no absolute prescription for devising and undertaking analysis of sociomaterial considerations. At any one time, the social and the material are co-defined [3]. Nevertheless, digital technologies (as design artifacts) can be taken apart and re-assembled to convey different material properties and thus different sociomaterial realities [4]. Our case study confirms this conclusion and provides justification for what is possible, as well as what is desirable but not feasible, with the current version of DIAMOUSES. It also suggests pathways in which systems such as DIAMOUSES can be isolated and re-configured to create new opportunities for remediated practices [18].

Thus, in the light of our findings, it stands to argue that sociomateriality brings to design several concerns and challenges, including the compelling need for (a) devising the appropriate mix of flexible representations to be embedded in technology; (b) inscribing imbrications of representations to smooth out discontinuities in practice, and (c) tracing use to assess the socio-material aspects of representational practices in virtual space. Through this lens, it is possible for each representation to zoom-in on some selected elements of practice which become the focus of attention, while others are ignored or pushed into the background. At the same time, imbrications of (different) representations undertake to establish the new virtuality which dissolves ambiguities and/or breakdowns and re-orients users' practice.

\section{REFERENCES}

[1] W. J. Orlikowski (2007). "Sociomaterial Practices: Exploring Technology at Work.", Organization Science, Organization Studies 28(9), pp. 1435-1448.
[2] R. Zammuto, T. Griffith, A. Majchrzak, D. Dougherty and S. Faraj (2007). "Information technology and the changing fabric of organization," Organization Science 18(5), pp. 749-762.

[3] W. J. Orlikowski and S. V. Scott (2008). "Sociomateriality: Challenging the separation of technology, work and organization" The Academy of Management Annals, 2(1), pp. 433-474.

[4] P. M. Leonardi (2013)."'Theoretical foundations for the study of sociomateriality", Information and Organization 23, pp. 59-76.

[5] M. Aanestad and T. Bratteteig Eds. (2013). Nordic contributions in IS Research, LNBIP 156, Springer-Verlag Berlin Heidelberg.

[6] A. Johri (2011). "Sociomaterial bricolage: The creation of locationspanning work practices by global software developers". Information and Software Technology 53 (9), pp. 955-968.

[7] L. Suchman (2007). Human-Machine Reconfigurations: Plans and Situated Actions, (2nd ed.), New York: Cambridge University Press.

[8] P. Dourish and M. Mazmanian (2012). Media as material: Information Representations as Material Foundations for Organizational Practice. In Paul Carlile, Davide Nicolini, Ann Langley and Haridimos Tsoukas (eds.) Perspectives on Process Organization Studies: How Matter Matters: Objects, Artifacts and Materiality in Organization Studies. Volume 3. Eds. Oxford University Press.

[9] E. Parmiggiani and M. Mikalsen (2013). "The Facets of Sociomateriality: A Systematic Mapping of Emerging Concepts and Definitions". In M. Aanestad and T. Bratteteig (Eds.): SCIS 2013, LNBIP 156, pp. 87-103, Springer-Verlag Berlin Heidelberg.

[10] D. Akoumianakis (2013). "Socio-materiality of online music ensembles: An analysis based on cultural artifacts \& affordances", The Science and Information Conference 2013 (SAI'2013), IEEE Computer Society.

[11] T. M. Harrison and B. Barthel (2009). "Wielding new media in Web 2.0: exploring the history of engagement with the collaborative construction of media products", New media \& Society, 11 (1\&2), pp. 155-178.

[12] K-B., Jensen and R. Helles (2011). "The internet as a cultural forum: Implications for research", New Media \& Society, 13, pp. 517-533.

[13] A. Gruzd, B. Wellman and Y. Takhteyev (2011): "Imagining Twitter as an Imagined Community", American Behavioral Scientist, 55(10), pp. 1294-1318.

[14] M. Zappavigna (2011). "Ambient affiliation: A linguistic perspective on Twitter", New media \& Society, 13 (5), pp. 788-806.

[15] D. Akoumianakis, N. Vidakis, A. Akrivos, G. Milolidakis, D. Kotsalis and G. Vellis (2011). "Building 'Flexible' vacation packages using collaborative assembly toolkits and dynamic packaging: The Case Study of the eKoNES", Journal of Vacation Marketing, 17(1), pp. 17-30.

[16] D. Akoumianakis and C. Alexandraki (2012). Collective Practices in Common Information Spaces: Insight from Two Case Studies, HumanComputer Interaction, 27 (4), pp. 311-351.

[17] J.-F. Blanchette (2011). A Material History of Bits. Journal of the American Society for Information Science and Technology, 62(6), pp. 1024-1057.

[18] D. Akoumianakis, G. Ktistakis, G. Vlachakis, P. Zervas and C. Alexandraki (2013). Collaborative music making as 'remediated' practice: Blending boundary objects and cloud services, Proc. of the 18th International Conference on Digital Signal Processing (DSP2013), Santorini, Greece, IEEE Press.

[19] P. H. Gochenour (2006). "Distributed communities and nodal subjects", New Media \& Society, 8(1), pp. 33-51.

[20] W. Kim, O-P. Jeong and S-W. Lee (2010). "On social web sites", Information Systems, 35, pp. 215-236.

[21] R. T. Schatzki, K. Knorr-Cetina, K. and E. von Savigny (2001). The Practice Turn in Contemporary Theory, pp. 1-14. London: Routledge.

[22] P. M. Leonardi (2011). When flexible routines meet flexible technologies: Affordance, constraint, and the imbrication of human and material agencies. MIS Quarterly, 35(1), pp. 147-167.

[23] D. L. Introna and N. Hayes (2011). On sociomaterial imbrications: What plagiarism detection systems reveal and why it matters. Information and Organization 21, pp. 107-122. 
[24] G. F. Lanzara (2010). "Remediation of practices: How new media change ways we see and do things in practical domains", First Monday, 15(6-7).

[25] J. Kallinikos, A. Aaltonen and A. Marton (2010). "A theory of digital objects", First Monday, 15 (6-7).

[26] D. E. Bailey, P. M. Leonardi and S. R. Barley (2012). "The Lure of the Virtual”, Organization Science 23(5), pp. 1485-1504.

[27] W. J. Orlikowski (2000). "Using Technology and Constructing Structures: A Practice Lens for Studying Technology in Organizations", Organization Science, 11(4), pp. 404-28.

[28] U. Schultze and W. Orlikowski (2004). "A Practice Perspective on Technology-Mediated Network Relations: The Use of Internet-Based Self-Serve Technologies", Information Systems Research, 15 (1), pp. 87-106.

[29] D. Akoumianakis, D. (2010). "Tracing community life across virtual settlements", International Journal of Virtual Communities and Social
Networking, 2(4), pp. 51-63.

[30] A. Pickering (1993). "The mangle of practice: agency and emergence in the sociology of science", The American Journal of Sociology, 99(3), pp. 559-589.

[31] J. Gibson (1979). The Ecological Approach to Visual Perception. Boston: Houghton Mifflin.

[32] D. A. Norman (1988). The Psychology of Everyday Things. New York: Basic Books

[33] D. A. Norman (1998). The Invisible Computer. Cambridge: MA, MIT.

[34] W. W. Gaver (1991). "Technology affordances", Proc. of the Annual Conference on Human Factors in Computing Systems (CHI'91), pp. 7984, ACM Press.

[35] C. Alexandraki, and D. Akoumianakis (2010). "Exploring New Perspectives in NMP: The DIAMOUSES Framework", Computer Music Journal, 34, pp. 66-83. 